\title{
Spectrum Simulation in DTSA-II
}

\author{
Nicholas W.M. Ritchie \\ National Institute of Standards and Technology, Gaithersburg, MD 20889-8371, USA
}

\begin{abstract}
Spectrum simulation is a useful practical and pedagogical tool. Particularly with complex samples or trace constituents, a simulation can help to understand the limits of the technique and the instrument parameters for the optimal measurement. DTSA-II, software for electron probe microanalysis, provides both easy to use and flexible tools for simulating common and less common sample geometries and materials. Analytical models based on $\varphi(\rho z)$ curves provide quick simulations of simple samples. Monte Carlo models based on electron and X-ray transport provide more sophisticated models of arbitrarily complex samples. DTSA-II provides a broad range of simulation tools in a framework with many different interchangeable physical models. In addition, DTSA-II provides tools for visualizing, comparing, manipulating, and quantifying simulated and measured spectra.
\end{abstract}

Key words: Monte Carlo, simulation, EPMA, EDS, X-ray, electron

\section{INTRODUCTION}

One of the most novel and useful components of the original Desktop Spectrum Analyzer (DTSA) (Fiori et al., 2005) performs energy dispersive $X$-ray microanalysis spectrum simulation. This component generates highly realistic electron excited spectra for bulk samples of arbitrary composition under realistic measurement conditions. These simulated spectra could be used to estimate limits of detection for experiment optimization and for educational purposes. As useful as it is, the spectrum simulation in DTSA is limited to bulk samples. Some of the most compelling problems for spectrum simulation are the more challenging microanalytical problems-complex geometries like particles, films, inclusions, and analysis of trace constituents. DTSA-II was inspired by the original DTSA but represents an entirely new, redesigned code base. The spectrum simulation component in DTSA-II is capable of handling samples with arbitrarily complex geometries and provides a full set of tools for studying limits-of-detection-type problems.

The original DTSA uses a $\varphi(\rho z)$ model of the distribution of inner shell ionization as a function of depth in the sample (Scott et al., 1995; Goldstein et al., 2003). The distribution of ionizations determines the distribution of generated X-rays, which are then tracked to a simulated detector and recorded. DTSA-II provides a similar analytical model. However, DTSA-II also provides the option to perform Monte Carlo simulation of electron and X-ray transport in arbitrarily complex sample geometries using

Received April 8, 2009; accepted June 26, 2009

*Corresponding author. E-mail: nicholas.ritchie@nist.gov the NISTMonte algorithms (Ritchie, 2005). Monte Carlo models of electron and X-ray transport simulate the trajectories of individual electrons and $\mathrm{X}$-rays as they pass through a sample and ultimately to the X-ray detector. In addition to generating realistic X-ray spectra, the Monte Carlo models in DTSA-II can also generate electron trajectory images, X-ray emission images, $\varphi(\rho z)$ curves, and other types of summary data. These output mechanisms are useful pedagogical tools for predicting and understanding the consequence of choices of experimental parameters. Details of DTSA-II's implementation of X-ray generation, X-ray transport, and X-ray detection for both the $\varphi(\rho z)$-based and Monte Carlo-based algorithms are discussed in this article.

In overview, DTSA-II is an application for X-ray microanalysis with a focus on quantification and simulation of energy dispersive X-ray spectra. DTSA-II focuses on rigorous standards-based quantification and simulation of unusual sample types and geometries. DTSA-II has been developed in Java ${ }^{\text {a }}$ for a high level of platform independence. It is being developed on Ubuntu (Debian-based) Linux, Microsoft Windows, and Apple OS X. DTSA-II has been carefully designed to keep the implementation of the algorithms independent of implementation of the user interface. Microanalytical algorithms and common shared user interface components are implemented in a library format (epq.jar), and glue that binds the algorithms into a user-friendly application is implemented in a separate mod-

${ }^{\mathrm{a}}$ Certain products, both public domain and commercial, are identified herein to provide adequately documentation. Identification of such products does not imply recommendation or endorsement by NIST, nor does it imply that the identified products are the best available. Conversely, the omission of any such products does not imply its unsuitability for use. 
ule (dtsa2.jar). ${ }^{\text {b }}$ This separation ensures that the algorithms can be readily reused independent of the DTSA-II application for purposes beyond those envisioned by the author.

\section{Models And Methods}

DTSA-II relies heavily on its ability to model X-ray detectors for both quantification and simulation. When measured spectra are read from disk into DTSA-II, the software encourages the analyst to associate a user-defined detector model. The detector model contains more detector performance parameters than are strictly necessary to quantify the spectra, but the additional information makes generating directly comparable simulations of measured spectra straightforward, robust, and reliable. The DTSA-II detector model is conceptually very general. An X-ray detector is simply something that accumulates energy and intensity data from a stream of simulated X-rays. A model can be implemented to simulate an ideal detector (quantum efficiency $=1.0$ at all energies), a realistic energy dispersive spectrometer (EDS) detector, or potentially even, if demand continues, a wavelength dispersive detector. While the ideal detector may be interesting and informative and is almost trivial to implement, realistic models of EDS detectors are more important for comparing simulated and measured spectra. The two dominant types of EDS in the field today are the lithium-drifted silicon detector $(\mathrm{Si}(\mathrm{Li}))$ and the silicon drift detector (SDD). A third much rarer class, microcalorimeters, is sufficiently interesting to merit consideration, and DTSA-II implements a simple microcalorimeter model.

From the perspective of a phenomenological model, $\mathrm{Si}(\mathrm{Li})$ and SDD are very similar (Knoll, 2000). Both detect $\mathrm{X}$-rays via conversion into electron/hole pairs in a block of ultra-high purity intrinsic silicon. The average number of electron/hole pairs generated is proportional to the X-ray energy with the conversion efficiency being $3.76 \mathrm{eV}$ per pair at $77 \mathrm{~K}$ and $3.62 \mathrm{eV}$ per pair at $300 \mathrm{~K}$. The actual number of generated electrons is distributed around the average with an approximately Gaussian distribution. The width of the Gaussian is less than would be predicted by Poisson statistics to an extent parameterized by the Fano factor, $F$. The electrons are swept out of the detection volume to an anode where the current pulse is collected and passed to a low noise current-to-voltage converter. The amplification and recording process introduces additional electronic noise that contributes to the final detector resolution. A simple expression for the Gaussian resolution of a $\mathrm{Si}(\mathrm{Li})$ or $\mathrm{SDD}$ detector as a function of X-ray energy is

\footnotetext{
${ }^{\mathrm{b}}$ Executable modules or libraries in Java are typically identified by the extension jar.
}

$$
\sigma(E)=\varepsilon \sqrt{n^{2}+\frac{E \cdot F}{\varepsilon}},
$$

where $\varepsilon$ is the conversion efficiency and $n$ accounts for the electronic noise. The Fano factor for silicon has been reported in the range 0.085 to 0.16 (Knoll, 2000), although measurements we have performed on several modern detectors suggest a value of $F=0.12$.

The thickness of the active area determines the fraction of impinging X-rays that are absorbed. The absorption efficiency may be calculated as a function of the X-ray energy from the density of silicon $\left(\rho=2.33 \mathrm{~g} / \mathrm{cm}^{3}\right)$, the thickness of the detector, and the mass absorption coefficient. The mass absorption coefficient is a critical parameter in many aspects of modeling X-ray transport. DTSA-II makes available several tabulations of measured, computed, and semiempirical mass absorption coefficients including the Henke 1993 tabulation (Henke et al., 1993), the National Institute of Standards and Technology (NIST) X-Ray Form Factor, Attenuation, and Scattering tables (Chantler et al., 2005), Heinrich's IXCOM-11 (Heinrich, 1986) semiempirical formula, and various other discrete tabulations of microanalytically interesting characteristic X-rays energies. The Henke and NIST tabulations are designed for interpolation with particular care taken around the discontinuities at absorption edges. The absorption of X-rays is governed by the Beer-Lambert law,

$$
I=I_{0} e^{-\mu \cdot \mathrm{t}}
$$

where $I_{0}$ is the incident intensity, $\mu=[\mu / \rho] \rho$ is the mass attenuation coefficient, and $t$ is the thickness of the absorber. The mass absorption coefficient is tabulated on an elemental basis, and mass absorption coefficients for compounds may be computed according to the following rule,

$$
\mu=\sum_{i=1}^{n} W_{i} \cdot \mu_{i}
$$

where $W_{i}$ and $\mu_{i}$ are, respectively, the weight fraction and mass attenuation coefficient for element $i$ in a compound with $n$ elements. This approximation neglects any chemical, molecular, or crystalline modifications to $\mu$. However, since the absorption typically involves ionization of a nonvalence electron, these modifications are typically small except just above absorption edges and at low energies $(<100 \mathrm{eV})$. Multilayer absorbers can be modeled through repeated application of equation (2).

It is worthwhile to make the distinction between mass attenuation coefficients and mass absorption coefficients clear. An energetic X-ray may interact with matter via photoabsorption, Compton scattering, or Rayleigh scattering. Photoabsorption involves the annihilation of the original X-ray and the ejection of an atomic electron resulting in 

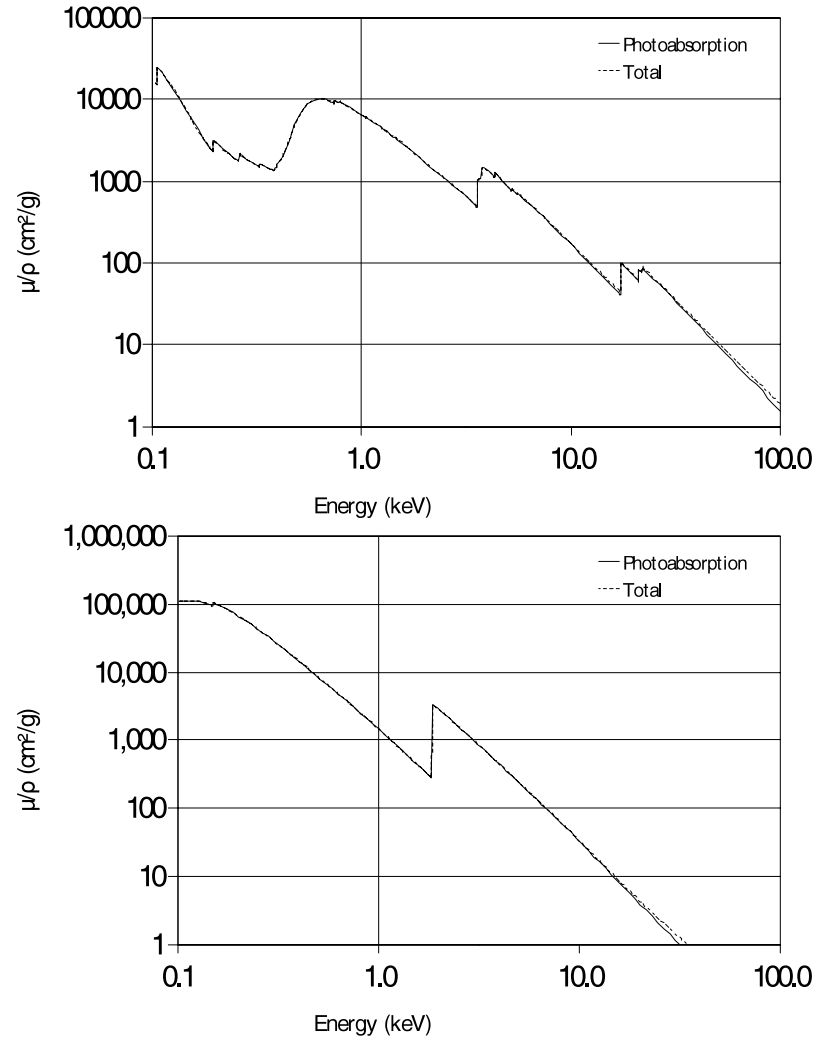

Figure 1. Comparing the mass absorption coefficient (photoabsorption) with the mass attenuation coefficient (photoabsorption + Compton + Rayleigh) for (a) silicon and (b) uranium (Chantler et al., 2005). Except at the highest energies, the mass attenuation coefficient is dominated by the mass absorption coefficient.

an ionized atom. The ionized atom is unstable and will relax via one or more of the standard mechanisms-Auger production, X-ray production, and Coster-Kronig transition. Compton scattering involves the deflection of the X-ray trajectory via scattering off of an unbound, slow-moving electron. Some of the incident X-ray energy is transferred to the electron so that the outgoing X-ray has less energy than the incident. Rayleigh scattering is the elastic (no energy loss) coherent scattering of X-rays from bound electrons. Figure 1 compares the mass absorption coefficient to the mass attenuation coefficient for silicon and uranium. The mass attenuation coefficient is dominated by mass absorption at the energies of interest to microanalysts. Artifacts due to Compton scattering are not typically observed in electron-excited X-ray spectra.

A fraction of the X-rays that are absorbed by the detector are reemitted as Si-K X-rays. The fraction is determined by the fluorescence yield for $\mathrm{Si}-\mathrm{K} \mathrm{X}$-rays $\left(\omega_{K}=\right.$ 0.055). A reemitted $X$-ray may be reabsorbed or may escape the detector. If it escapes the detector, it reduces the total number of electrons generated by an amount equivalent to a Si-K X-ray $(1.74 \mathrm{keV})$ and produces an artifact called an escape peak. Escape peaks are only possible for X-rays with energies above the $\mathrm{Si}-\mathrm{K}$ absorption edge $(1.838 \mathrm{keV})$. Escape peaks are more prominent in smaller area and thinner detectors.

Most silicon detectors are produced through a process of compensation with drifted lithium ions. This process can result in an inactive (or, more precisely, a partially active) layer on the entrance face of the detector. This inactive region is referred to as the dead layer. Some X-rays will be absorbed by this layer, but they will produce fewer recorded electron/hole pairs than the same energy X-ray absorbed in the active area. $\mathrm{X}$-rays that are strongly absorbed by silicon are affected the most by the dead layer. The dead layer has two principal effects on the measured spectrum. First, the dead layer acts like a thin silicon window filtering out a fraction of the incident X-rays before they reach the active measurement volume. The window effect can be computed from an estimate of the thickness of the dead layer using equation (2). Second, the dead layer causes an effect called incomplete charge collection. Incomplete charge collection is observed as additional counts enhancing the intensity on the low energy side of characteristic X-ray lines. An X-ray absorbed in the partially active layer will contribute fewer than $E / \varepsilon$ electron/hole pairs per X-ray. Incomplete charge collection is often modeled as an exponential tail on the low energy side of characteristic peaks. Detector manufactures attempt to minimize the dead layer thickness to enhance low energy sensitivity and to optimize peak shape. At least one SDD manufacturer (Niculae et al., 2008) seems to have all but eliminated incomplete charge collection though a proprietary process that makes the dead layer truly dead rather than simply partially inactive.

Additional surface layers typically of nickel, gold, or aluminum are deposited on the surface of the detector as an electrode to produce the fields necessary to sweep the electron/hole pairs from the active volume to the detection anode.

The efficiency of the active layer is $1-\exp \left(-\mu_{S i} \cdot t_{d e t}\right)$, where $t_{\text {det }}$ is the thickness of the active layer.

While historically some detectors were designed without entrance windows, almost all detectors have some kind of vacuum tight window separating the sample chamber and the detector crystal. Most modern detectors use a polymer window or occasionally, when low energy performance is not critical, a beryllium window. Polymer windows typically use a silicon support structure with approximately 70 $80 \%$ open area and a thin aluminum coating to provide light tightness. Windows can be modeled using equations (2) and (3) when the composition and thickness of the window are known. The effect of the silicon grid can be modeled by noting that it only obstructs a fraction of the incident X-rays and becomes increasingly transparent above about $10 \mathrm{keV}$. DTSA-II makes available a range of common window types, and others can be easily added by modifying and recompiling the source code.

In most cases, DTSA-II models the transmission of the windows, coatings, and dead layers through repeated appli- 
cation of the Beer-Lambert equation. Our ability to model windows is ultimately limited by our knowledge of the composition, thickness, and layer structure and of the mass absorption coefficients. In one case (Moxtek AP 3.3), the window manufacturer provides tabulated nominal transmission tables. Both calculated and tabulated windows models are implemented for the AP 3.3.

The resolution performance of the detector is modeled by convolving the recorded X-rays with a normalized peak shape function. Nominally, the peak shape function is Gaussian with width given by equation (1). Poisson count statistics (Press et al., 1992) may be applied channel-by-channel to the resulting spectrum.

In addition to models that simulate common laboratory detectors, it is also possible to create detector models that record X-ray intensities in ways that are difficult to realize with laboratory devices. For example, laboratory detectors cannot readily record the spatial location at which an X-ray is generated. This information is available in the Monte Carlo and can be used to implement detectors that record the $\varphi(\rho z)$ curve or that create images showing the spatial distribution of emitted X-rays. Furthermore, the modeled detectors need not be limited in resolution and can tabulate lines on a line-by-line basis and can differentiate bremsstrahlung from characteristic X-rays. The graphical user interface (GUI) to DTSA-II tabulates generated and emitted intensities and emitted intensity images as part of the standard HTML report.

\section{Electron Transport and X-Ray Generation and Transport}

The Monte Carlo simulation in DTSA-II is built on electron transport algorithms in NISTMonte. A detailed description of the NISTMonte Monte Carlo simulation of electron transport in arbitrary geometry samples is provided elsewhere (Ritchie, 2005; Villarrubia et al., 2007). NISTMonte/ DTSA-II builds arbitrary shaped geometries from a series of simple primitive shapes including spheres, cylinders, and volumes enclosed by planes. The sum or difference of primitive shapes may be joined to form more complex shapes. Furthermore, shapes can be embedded within shapes to simulate inclusions. Shapes are filled with materials that are defined by elemental weight percent and density. There are no arbitrary limits to the number of elements in a material or in a model. DTSA-II provides a default set of simple sample geometries through the GUI. It is also possible to simulate more complex geometries through a scripting interface.

Electron energy loss is modeled using the continuous slowing down approximation. This approximation, which models the average affect of many small energy loss events, is most appropriate for energies well above the binding energies of valence electrons. Since X-ray simulation involves tracking the electron energies down to the lowest relevant edge energy, this approximation is almost invari- ably reasonable. Otherwise inelastic scattering processes are not modeled. Energy loss to core ionization or bremsstrahlung production, although producing large changes in electron energy, is sufficiently rare that it may be ignored as a mechanism for electron energy loss. Elastic scattering can be modeled using an analytical screened Rutherford cross section (Myklebust et al., 1976) or various different tabulated Mott cross sections (Czyzewski et al., 1990; Jablonski et al., 2003). By default, DTSA-II uses the NIST Electron Elastic-Scattering Cross-Section Database-Version 3.1 and the Joy-Luo (Joy \& Luo, 1989) variant of the Bethe energy loss model. Switching this and most other algorithms is easy through the scripting interface.

Energetic electrons in matter create X-rays through one of two processes - characteristic X-ray generation and bremsstrahlung production. DTSA-II models these independently facilitating the study of one, the other, or both. Characteristic X-rays are modeled in a class called XRayEventListener2, and bremsstrahlung is modeled in a class called BremsstrahlungEventListener. The suffix EventListener in the class names suggests how these classes fit within the library architecture. EventListener classes work in partnership with classes that are sources of event notifications. An EventListener classes register with an event source class to be notified whenever an interesting event occurs. The MonteCarloSS class acts as an event source and provides notifications whenever electrons scatter or one of about half-a-dozen other interested events occur during electron transport. The XRayEventListener2 and BremsstrahlungEventListener classes watch the MonteCarloSS class waiting for elastic scattering events. Then these two classes perform the necessary calculations to simulate X-ray generation. Depending upon the information desired from the simulation, either an XRayEventListener2 or a BremsstrahlungEventListener or both or neither may be attached to the MonteCarloSS instance. This flexibility enhances efficiency as only the required information is computed each time. Similarly one or more detectors may be attached to the XRayEventListener2 or BremsstrahlungEventListener to tabulate the desired information. The X-ray detector models work in a similar manner. They too act like EventListener objects except that now the XRayEventListener2 and/or the BremsstrahlungEventListener classes act as event sources and provide notification of X-ray generation related events. Multiple EventListener-type classes can attach to a single event source.

At each elastic scattering event, the XRayEventListener2 and the BremsstrahlungEventListener compute the likelihood of an X-ray emission in the intervening path segment. The emission is assumed to have been generated at a point selected at random between the previous scattering point and this. The emission is then tracked through the intervening material to the detector and recorded. Absorption in the intervening material is modeled by tabulating the mass thickness of each material between the point of emission and the detector and computing the transmitted fraction. Tabulating the mass thickness is relatively expen- 
sive and must be performed once per scattering/generation event.

The intensity of generated characteristic X-rays in photons per differential unit of electron path $(d s)$ is

$$
d I_{i A}=\frac{C_{A} \rho N_{A}}{A_{A}} p_{i A} Q_{A}(E) d s,
$$

where $C_{A}$ is the amount of element $A$ with atomic weight $A_{A}$ in weight percent, $N_{A}$ is Avagadro's number, $\rho$ is the density, $p_{i A}$ is the probability that an ionization will decay via the $i$ 'th characteristics X-ray transition, and $Q_{A}(E)$ is the cross section for ionization of a shell that may lead to a decay via the $i$ 'th characteristic transition transition by an electron with energy $E$. The term $p_{i A}$ encompasses both the fluorescence yield and the relative weights of lines due to characteristic and Coster-Kronig transitions. Equation (4) is fairly trivial to implement in a Monte Carlo. Each time the electron scatters, the intensity generated in the previous trajectory segment is computed. The differential unit of path $d s$ is the distance between elastic scatter events. To eliminate bias, the generation is assigned to a randomly selected point located between this scattering point and the previous. The material in which the electron is propagating determines the elements, the weight fractions, and the density. $d I_{i A}$ is computed at each scattering event for each possible transition from each shell capable of being ionized for each element present in the sample. Equation (4) can be evaluated relatively efficiently by organizing the transitions by shells and realizing that much of the expression depends upon the element and shell. Only the $p_{i A}$ term must be evaluated for each transition.

$d I_{i A}$ represents the generated intensity. The intensity is assumed to be emitted isotropically and only that intensity which is emitted directly into the solid angle subtended by the detector is detected. A ray is drawn from the generation point toward the center of the detector. This ray is broken into segments, each representing passage through one intervening material. The transmission is computed for each segment, and the resulting net transmitted intensity is computed and recorded on the detector for each characteristic $\mathrm{X}$-ray at each scattering event.

DTSA-II provides two expressions for the cross section for ionization of a neutral atom by an energetic electron $Q_{A}$-Casnati et al. (1982) and Bote and Salvat (2008).c Casnati is a semiempirical expression based on measurements of the K-shell ionization cross section and extrapolated to the $\mathrm{L}$ and $\mathrm{M}$ shells. Bote/Salvat cross sections are analytical expressions fit to tabulations of distorted Born

\footnotetext{
${ }^{\mathrm{c}}$ The tabulations in this article have been summarized as parameterized equations. This parameterization, which the author received through personal communication, is implement in DTSA-II. A forthcoming article by D. Bote, F. Salvat, A. Jablonski, and C. Powell will detail the parameterization.
}

plane wave calculations. The Bote/Salvat cross section is the more modern and presumably more accurate expression and is thus the default.

The ionization cross section quantifies the probability of a neutral atom being ionized by an energetic electron. This probability integrated over a single electron trajectory is typically much less than one. Most electrons do not generate even a single X-ray. Modeling X-ray production by tracking ionizations and following $\mathrm{X}$-ray trajectories directly would require tracking as many electrons in simulation as in the equivalent experiment. For example, a $60 \mathrm{~s}$ acquisition at $1 \mathrm{nA}$ involves $3.7 \times 10^{11}$ electrons. It is not practical to directly simulate this number of electrons. Instead Monte Carlo simulations adopt various different strategies to optimize the calculation. PENELOPE (Salvat et al., 2006) enhances the number of events through a process called interaction forcing. Interaction forcing involves increasing the cross section for a mechanism by a forcing factor $(>1)$. Subsequent interactions must also be scaled to account for the forcing factor. The PENEPMA module of PENELOPE forces the interactions for ionization, bremsstrahlung production, X-ray absorption, and Compton scattering. Forcing naturally handles primary X-ray emission as well as secondary emission (fluorescence.) It is a sophisticated mechanism and, while more efficient than brute force, it remains slow. Only a very small fraction of emitted X-rays actually strike the detector. Simulations with Penelope typically take hours. However, secondary fluorescence is a natural consequence of the model.

DTSA-II takes a different approach. DTSA-II accumulates the probability of X-ray events rather than actual events. Each trajectory segment for each electron contributes to ionize each available shell and thereby contributes to the accumulated probability of X-ray generation for each available X-ray line. The accumulated probability is scaled by the ratio of the desired experimental electron dose divided by the number of simulated electrons. The result is, on average, the actual number of X-rays generated. Poisson statistics may be applied directly to the resulting spectrum.

DTSA-II's approach requires approximately 1,000 electron trajectories or less than a minute on a typical circa 2008 computer to generate a usable X-ray spectrum on bulk material. Thin films and small particles require more trajectories, but each trajectory takes less computation time. The DTSA-II approach has its downside. First, all count statistics information is lost and must be added back by assuming Poisson statistics on a channel-by-channel basis. Second, secondary processes such as fluorescence due to primary radiation are not naturally included in the model. Secondary fluorescence can be added back in, but currently DTSA-II does not model secondary fluorescence.

Following ionization, the atom will relax via X-ray production, Auger production, or Coster-Kronig transition. A single ionization may lead to multiple Auger and X-ray emissions as the atom cascades through a series of steps back to a neutral ground state. The relaxation process as 
encapsulated in the term $p_{i A}$ is modeled using tabulations extracted from ENDLIB97 (Cullen, 1992). For a given shell ionization, this tabulation provides the relative probability of each available characteristic X-ray line. The tabulation accounts for both the first transition that fills the ionized shell and subsequent transitions until the atom returns to the neutral ground state.

Bremsstrahlung is modeled by interpolating the tabulated bremsstrahlung production cross sections of Seltzer and Berger (1986). These element-by-element tabulations provide the partial cross section for the generation of a bremsstrahlung X-ray photon of a specified energy as a function of the electron energy. Integrating the partial cross section over the emitted photon energies gives the total cross section-the cross section for the production of a bremsstrahlung photon of any energy. For each trajectory segment and for each element in the current material, the probability of a bremsstrahlung photon of any energy is computed.

$$
d P_{B, A}=\frac{C_{A} \rho N_{A}}{A_{A}} Q_{B, A}(E) d s
$$

where $C_{A}, \rho, N_{A}, A_{A}$, and $d s$ are the same as in equation (4) and $Q_{B, A}(E)$ is the total cross section for bremsstrahlung production for the element $A$ as a function of electron energy. The total probability for the production of a bremsstrahlung photon of any energy from any element is

$$
d P_{B}=\sum_{A} d P_{B, A}
$$

To enhance the computational efficiency, one element is selected at random weighted by $d P_{B, A}$ to produce bremsstrahlung at each scatterpoint and the full probability $d P_{B}$ is assigned to that element. To ensure a smooth bremsstrahlung background, a small number of events $N_{B}$ are generated for the selected element, each with probability $d P_{B} / N_{B}$. The energy of each event is selected at random so that an ensemble of similar selections will produce the distribution specified by the partial cross section as a function of bremsstrahlung photon energy.

Bremsstrahlung production is not isotropic. The direction of the incident electron defines an axis about which emission is cylindrically symmetric. At low electron energies, energetic photons tend to be emitted in a dipole pattern. At low photon energies and high electron energies (approaching the rest energy of an electron), the distribution is enhanced in the direction of electron propagation. Kissel et al. (1983) tabulated the shape functions for atomic bremsstrahlung for selected atoms and electron energies. This tabulation is not easily interpolated and sampled so Acosta et al. (2002) reparameterized it in terms of a two parameter Lorentz transformed expression. DTSA-II implements the Acosta parameterization and scales the emitted intensity by the angle dependent probability according to the angle between the electron trajectory and a ray to the $\mathrm{X}$-ray detector. In practice, however, particularly at the energies typical of X-ray microanalysis, the difference between isotropic production and the correct angular distribution is not significant. The direction of the incident electron is quickly randomized thus randomizing the emission of bremsstrahlung photons. The difference in computational expense is high. Evaluating the Acosta distribution involves a three-dimensional $\left(E_{\text {electron }}, Z\right.$, and $\left.E_{B}\right)$ cubic spline interpolation, and the difference is not of critical important because bremsstrahlung intensity is rarely used for quantitative measurements. By default, DTSA-II computes the bremsstrahlung assuming an isotropic distribution, but switching distributions is easy through the GUI preferences. At $25 \mathrm{keV}$ on bulk $\mathrm{K} 412$, the isotropic model produces roughly the same shaped bremsstrahlung background with $13 \%$ more intensity. One would expect the effect would be larger in thin film samples where the electron trajectories are most uniformly oriented. At $25 \mathrm{keV}$ on $0.1 \mu \mathrm{m}$ of K412, the isotropic model produces a similar shaped background with about $50 \%$ more intensity. Figure 2 compares bremsstrahlung backgrounds computed using isotropic and Acosta, Llovet, and Salvat's distributions for bulk K412 at $30 \mathrm{keV}$.

It should be noted that the characteristic and bremsstrahlung production models are almost totally independent but taken together naturally produce spectra in which the ratio of characteristic to bremsstrahlung is typically accurate to much better than a factor of 2 . This fact alone is highly suggestive that both characteristic and bremsstrahlung radiation are modeled generally correctly.

\section{Analytical Simulations}

The analytical simulation is based on the same $\varphi(\rho z)$ curves used for quantitative correction of atomic number and absorption effects. The $\varphi(\rho z)$ curve is defined as the ratio of the number of ionizations at the mass depth $\rho z$ relative to the number of ionizations in an infinitesimally thin film. $\varphi(\rho z=0)$ invariably exceeds 1.0 because the first infinitesimal slice has contributions from both those electrons that are on their first passage through the slice (equal to 1.0 by definition) plus those that are backscattered from deeper in the bulk. The number of photons generated in the infinitesimal slice can be computed from equation (4) times the electron dose, $D=t_{L} I_{P} / q_{e}$, where $t_{L}$ is the live-time, $I_{P}$ is the probe current, and $q_{e}$ is the charge of a single electron. The number of X-rays generated in the differential slice of mass depth $d \rho z$ is

$$
d G(\rho z)=Q_{i A}\left(E_{0}\right) D\left(\frac{C_{A} N_{A} \rho}{A_{A}}\right) p_{i A} \varphi(\rho z) d \rho z,
$$

where the ionization cross section $Q_{i A}\left(E_{0}\right)$ is evaluated at the incident beam energy $E_{0}$ regardless of the average elec- 


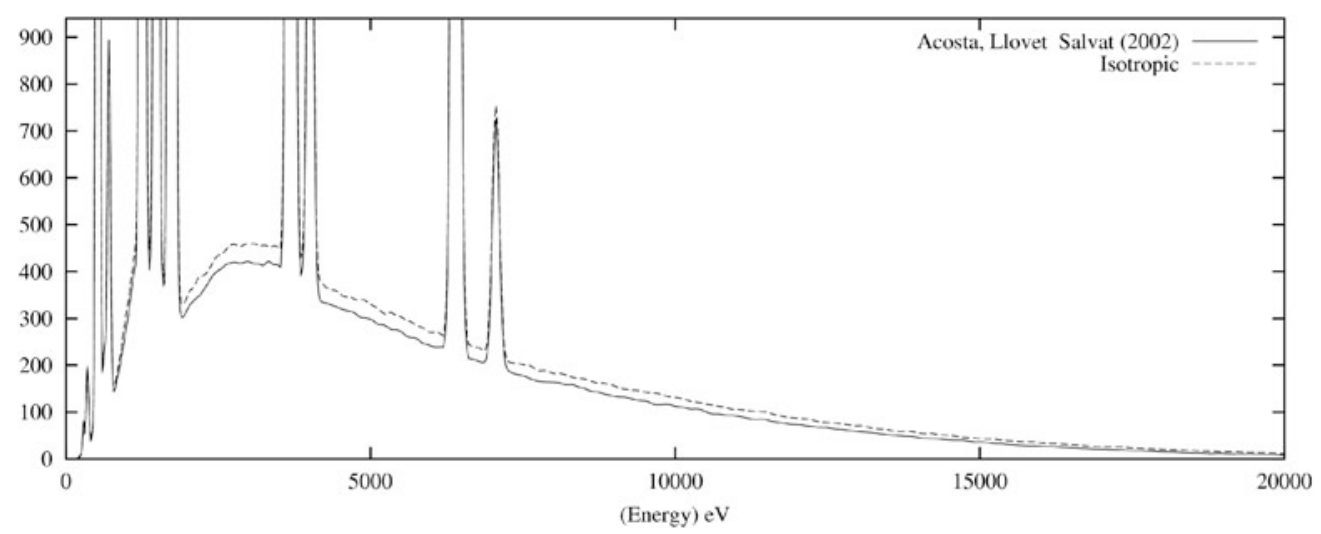

Figure 2. Comparing the shape and intensity of the bremsstrahlung as modeled using my implementation of Acosta et al.'s (2002) angular distribution and an isotropic distribution. The material is K412 glass, and the incident beam energy is $30 \mathrm{keV}$.

tron energy at depth $\rho z$. The total generated intensity can be computed by integrating equation (7) over $\rho z$.

The atomic number and absorption correction $F(\chi)$ is computed by integrating $\varphi(\rho z)$ times a factor to account for absorption over $\rho z$,

$$
F(\chi)=\int_{0}^{\infty} \varphi(\rho z) \exp (-\chi \rho z) d \rho z
$$

where $\chi=(\mu \rho) \sin (\theta)$ and $\theta$ is the take-off angle. The $Z \cdot A$ terms in the $Z \cdot A \cdot F$ correction equal the ratio of $F(\chi)$ computed for the unknown material and $F(\chi)$ compute for the standard material. Since $\varphi(\rho z)$ is a ratio and thus dimensionless, $F(\chi)$ has the dimension length.

The emitted intensity may be calculated from equation (7) by performing a similar integration:

$$
I_{i A}=Q_{i A}\left(E_{0}\right) D\left(\frac{C_{A} N_{A} \rho}{A_{A}}\right) p_{i A} F\left(\chi_{i A}\right) .
$$

The parameter $\chi_{i A}$ includes details about the position of the detector. The active area, distance, and efficiency of the detector determine the fraction of the emitted intensity that is measured.

DTSA-II implements PROZA-96 (Bastin et al., 1998) and PAP and XPP (Pouchou \& Pichoir, 1991). PROZA-96 and XPP support nonnormal beam incidence. $\varphi(\rho z)$ is parameterized by the material composition, the atomic shell, and the electron energy. It is worthwhile to note that sometimes $\varphi(\rho z)$ models are described as a model of X-ray generation as a function of depth for a specific X-ray line. This is not strictly correct, and the distinction is important when simulating spectra. The first step in the relaxation process of a $\mathrm{K}$ shell is a $\mathrm{K}$-shell characteristic $\mathrm{X}$-ray $\left(\mathrm{K}-\mathrm{L}_{\mathrm{III}}\right.$, $\mathrm{K}-\mathrm{M}_{\mathrm{III}}$, etc.) or K-shell Auger emission. It is possible, however, that this process may leave the atom with an $\mathrm{L}$ - or
M-shell ionization, which may relax via an L- or M-shell characteristic X-ray $\left(\mathrm{L}_{\mathrm{III}}-\mathrm{M}_{\mathrm{V}}, \mathrm{L}_{\mathrm{III}}-\mathrm{N}_{\mathrm{V}}, \mathrm{M}_{\mathrm{III}}-\mathrm{N}_{\mathrm{IV}}\right.$, etc.). Since an atom does not move significantly between emitting the first K-shell characteristic X-ray and the subsequent L-shell characteristic X-ray emission, the spatial distributions are identical. Admittedly the L- or M-shell characteristic X-ray intensity following a $\mathrm{K}$-shell characteristic X-ray is very small because $\omega_{L}, \omega_{M} \ll 1$, and to a very good approximation the overall distribution of X-ray emission is the same as the distribution of the principal characteristic X-ray emission. A similar argument can be made about L-shell ionization. This argument should not be misinterpreted as saying that the $\varphi(\rho z)$ curve is the same for K-, L-, and M-shell ionization.

Bremsstrahlung is computed using Small's analytical expression (Small et al., 1987). Small computes the absolute generated intensity per $10 \mathrm{eV}$ bin and is based on careful analysis of many pure elemental spectra. The detector position and character determine the measured intensity. No further scaling is necessary to match characteristic and bremsstrahlung intensities to produce realistic spectra.

The generated bremsstrahlung intensity at energy $E_{\nu}$, $I_{\nu}$, in Small's model

$$
I_{\nu}=10^{5} e^{B}[Z(U-1)]^{M}
$$

is parameterized in terms of $M$ and $B$, which are functions of beam energy $E_{0}$,

$$
\begin{gathered}
M=0.00599 E_{0}+1.05 \\
B=-0.0322 E_{0}+5.80,
\end{gathered}
$$

and the over-voltage $U=E_{\nu} / E_{0}$. The generated intensity is corrected for backscatter and absorption. The emitted intensity is recorded the same way as characteristic X-ray emission. 


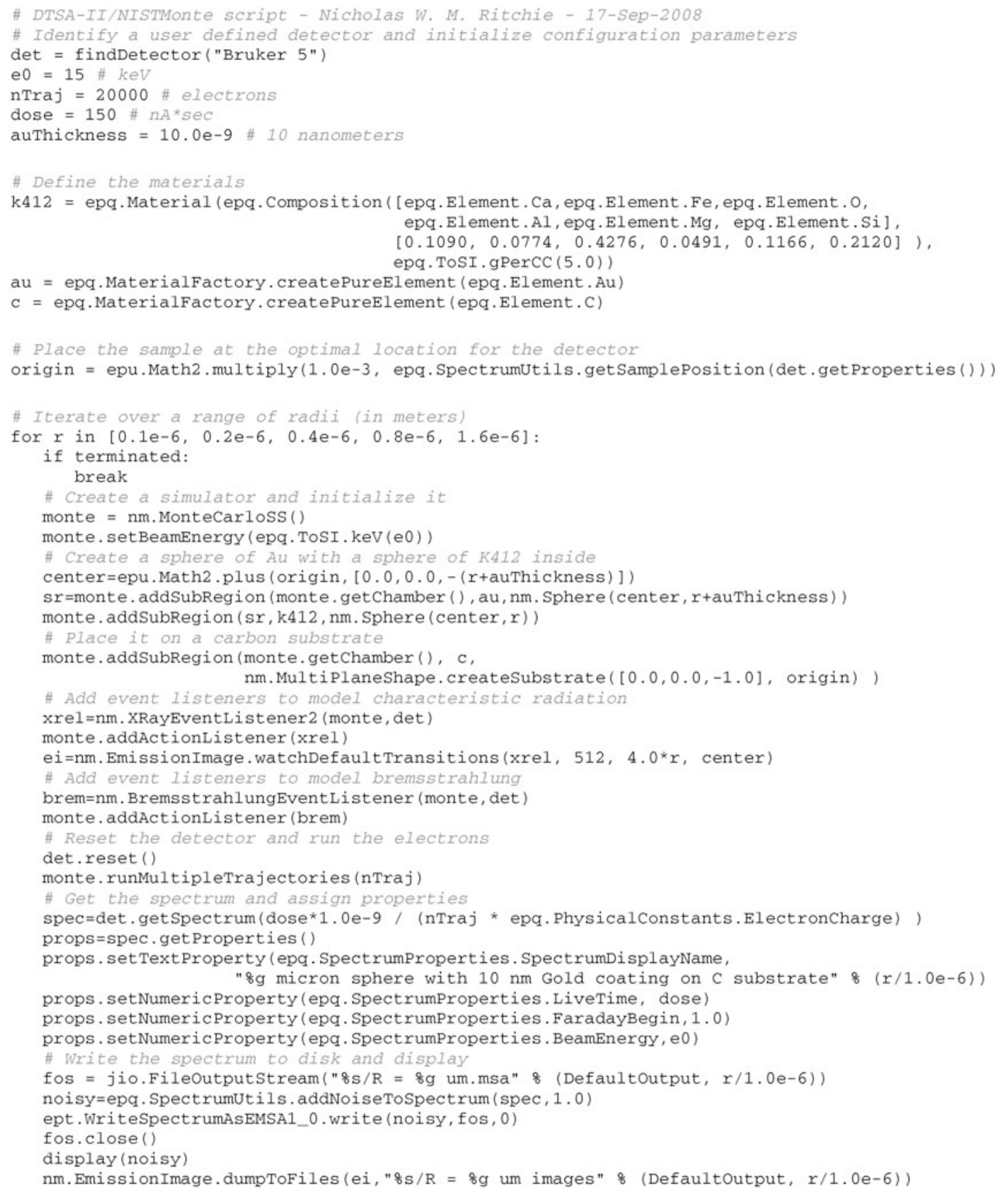

Figure 3. A basic script for generating a series of spectra and emission images for various sizes of K411 particles coated with $10 \mathrm{~nm}$ of gold and mounted on a carbon substrate. The code is in black type, and comments are in gray italics.

\section{Using the Simulators}

There are two distinct ways to interact with the spectrum simulation tools in DTSA-II. The easier to use is the GUI. This mechanism allows the user to generate simulated spectra for bulk samples using the analytical model or bulk, film on bulk, sphere on bulk, cube on bulk, or inclusion in bulk using the Monte Carlo model. In any of these models, the bulk may be a null material providing the equivalent of a free standing film, sphere, cube, or inclusion. The GUI leads you through the process of specifying a material or materials, specifying a detector and calibration, specifying instrument conditions, and specifying output options. You may select a noise-free spectrum or an arbitrary number of Poisson modified noisy spectra. The resulting spectrum or spectra are automatically saved to an EMSA file (Edgerton et al., 1991) and made available through the spectrum visualization and manipulation tools. In addition, an HTML report is generated summarizing the simulation parameters and the results including a virtual reality markup language 


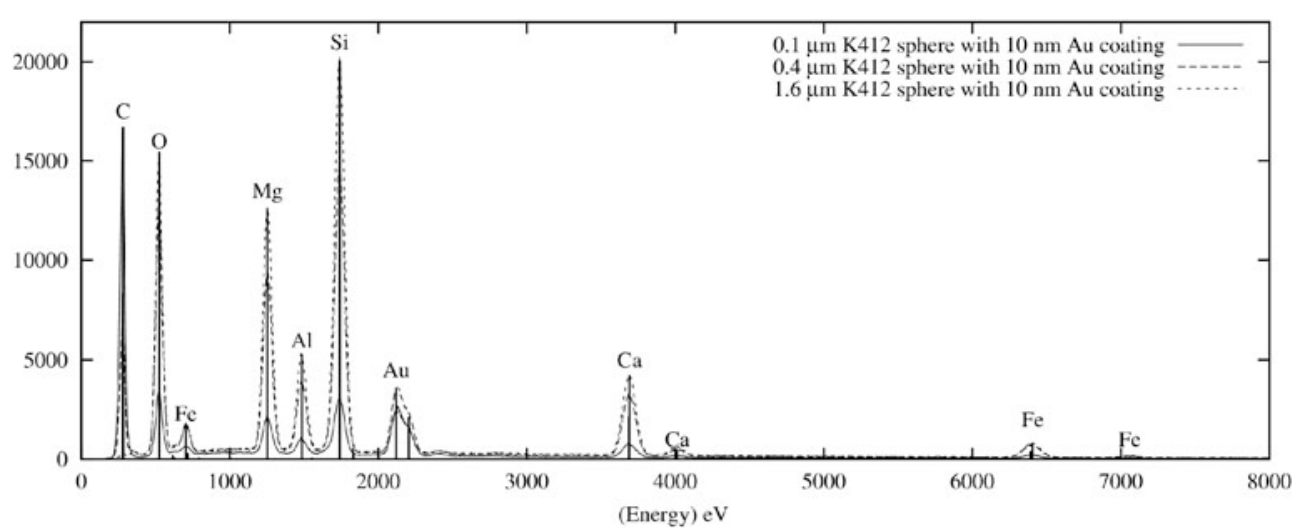

Figure 4. Three of the five spectra produced by the script in Figure 3. The spectra represent simulated K412 spheres of various radii coated with $10 \mathrm{~nm}$ of gold and mounted on a carbon substrate.

visualization of electron trajectories, a table of generated and emitted intensities (Monte Carlo only), and emitted intensity images (Monte Carlo-only option.)

The GUI mechanism is easy to use but limited. The Monte Carlo is capable of simulating much more complex geometries and providing additional information through the scripting interface. Scripting is an integral part of the DTSA-II user interface. Through scripting, it is possible to access all the algorithms available through the DTSA-II GUI plus many additional. Scripting is implemented through the Jython (http://www.jython.org) open-source library. Jython is a Java implementation of the Python language, a popular object-oriented scripting language. Jython has access to the full Java library set plus all the algorithms and data tabulations in DTSA-II and the Electron Probe Quantification (EPQ) library.

Figure 3 demonstrates a script for generating a series of spectra and emission images from a sphere of K412 of various radii coated with $10 \mathrm{~nm}$ of gold resting on a carbon substrate. The resulting spectra and three emission images are shown in Figures 4 and 5. This script demonstrates how scripting can be used to simulate geometries that are not supported by the GUI or to automate simulation of a range of parameters. This script can be readily modified to simulate other geometries, to use other detectors, or to iterate through other parameter sets. The EPQ library is documented in HTML at http://www.cstl.nist.gov/div837/837.02/ epq/dtsa2/JavaDoc/index.html using JavaDoc (http://java. sun.com/j2se/javadoc/).

\section{Results}

The NIST certified composition of K412 glass (SRM-470) is $10.90 \% \pm 0.14 \% \mathrm{Ca}, 42.76 \% \pm 0.18 \%$ O, $7.74 \% \pm 0.16 \%$ $\mathrm{Fe}, 4.91 \% \pm 0.11 \% \mathrm{Al}, 11.66 \% \pm 0.12 \% \mathrm{Mg}, 21.20 \% \pm$ $0.09 \%$ Si by mass fraction. The remaining $0.83 \% \pm 0.30 \%$ is unspecified. The input for the simulations is the certified composition normalized to $100 \%$. At $15 \mathrm{keV}$, the dominant lines for each of these elements result from K-shell ionizations. Thus this sample makes a good test sample to demonstrate the ability to simulate spectra in which all the major lines are restricted to the same family. Figure 6 shows a K412 measured on a Bruker 4040 SDD detector mounted on a JEOL JXA-8500F compared to Monte Carlo and analytically simulated spectra. Both the Monte Carlo and the analytical simulations are designed to compute the absolute measured spectral intensity for both bremsstrahlung and characteristic radiation from the basic models for ionization, relaxation, and detection. There are no gross arbitrary scaling factors. However, in practice, a small global scaling factor is needed to precisely match measured spectra. The scaling factor can be selected to match the intensity at a single energy, the integrated intensity, or some other global metric. The need for a scaling factor can be attributed to poor knowledge of the physics and/or the detector geometry. This scaling factor can either be implemented as a fudge factor or by changing the sample/detector distance to match simulated and measured intensities.

All the simulated spectra presented in this article have been scaled by changing the effective detector distance to match the Si K intensity between the measured K412 and the Monte Carlo simulated K412. Si K was chosen because it is an intense peak and of sufficiently high energy that the detector efficiency had effectively saturated. The nominal distance from the detector crystal to the sample is estimated to be $67 \mathrm{~mm}$, but it was found that using a distance of $72 \mathrm{~mm}$ resulted in a match of the Si intensities. The detector parameters for the Monte Carlo and analytical simulations are presented in Table 1 .

Table 2 summarizes the ratios of the simulated and measured intensities in the $\mathrm{K}$ or $\mathrm{K} \alpha$ line for each of the elements in K412 glass. In both the Monte Carlo and analytical simulations, the oxygen intensity is over $20 \%$ too low. This could be due to an underestimate of the efficiency 

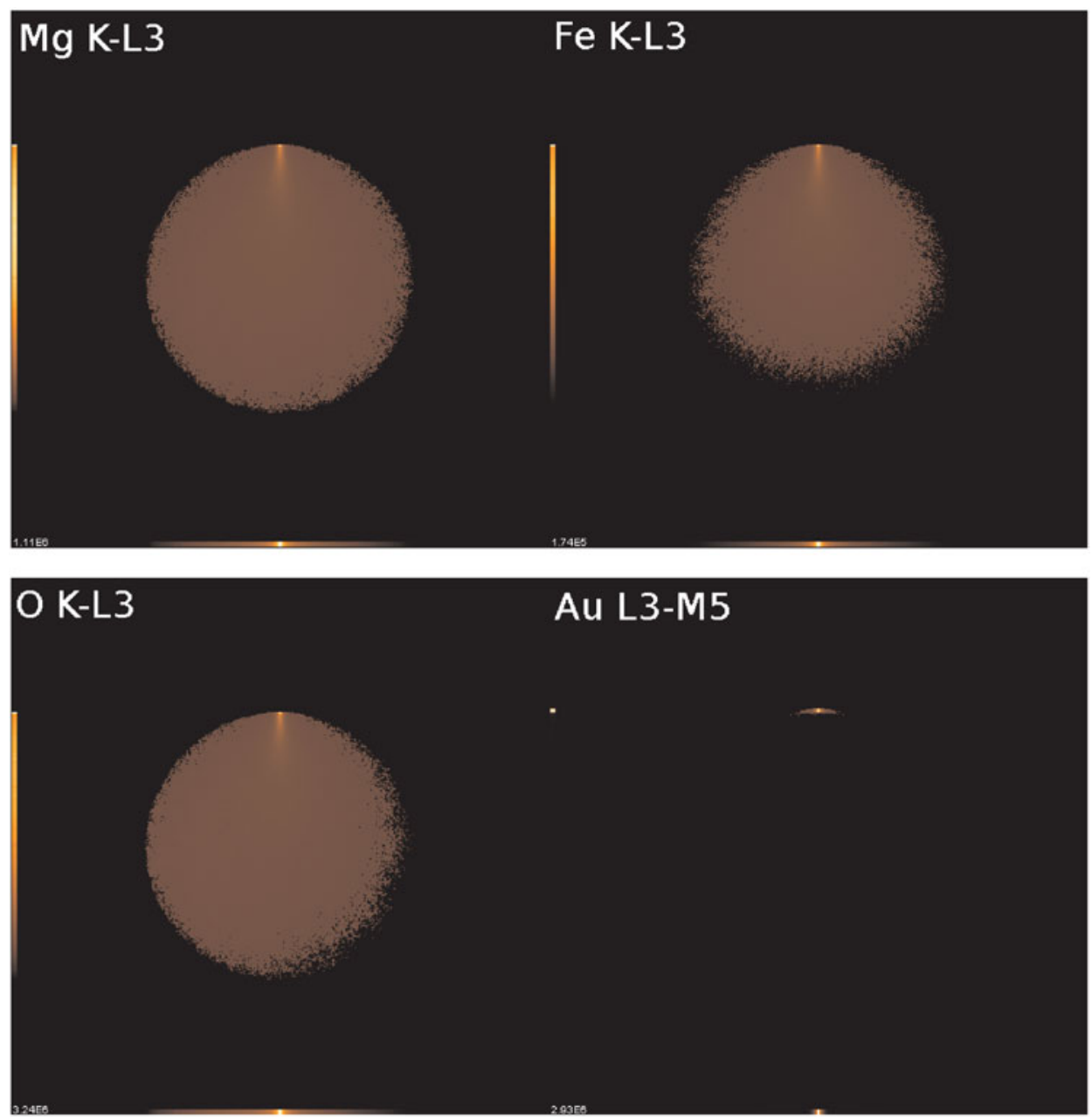

Figure 5. Four emission images create by the script in Figure 3. These images represent the $0.4 \mu \mathrm{m}$ radius sphere of K412 with a $10 \mathrm{~nm}$ coating of gold. You can see the interplay of overvoltage and absorption in these images. The image representing $\mathrm{Mg} \mathrm{K}$-L3 shows that at high overvoltage $(\sim 11)$ and sufficiently high energy $(1.3 \mathrm{keV})$ the measured X-rays come from throughout the particle. Fe K-L3, which has a lower overvoltage $(\sim 2.1)$, shows that the excitation volume diminishes in size. O K-L3 has sufficient overvoltage ( $\sim 28)$ but its emission in the right lower side (farthest from the detector) is absorbed preferentially before it can reach the detector. The Au L3-M5 image shows a little emission localized where the beam enters the sphere from the $10 \mathrm{~nm}$ coating of gold.

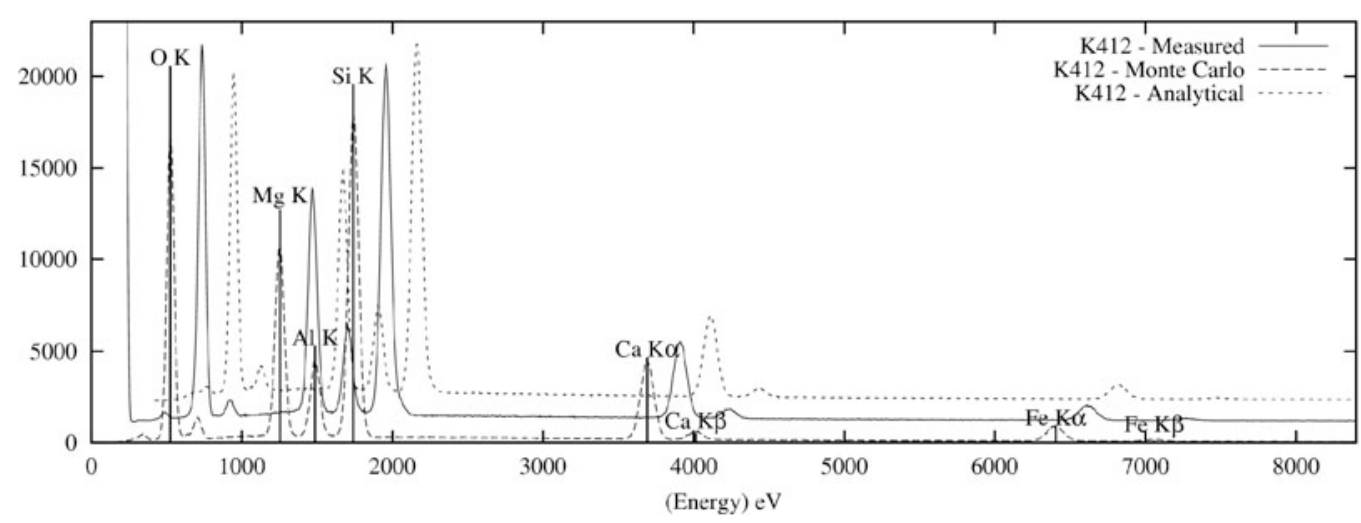

Figure 6. Compares Monte Carlo simulated, analytically simulated, and measured spectra from K412 glass at $15 \mathrm{keV}$. These spectra compare simulation with measurement for a sequence of $\mathrm{K}$ lines from $\mathrm{O}$ to Fe. The relative intensities are compared in Table 2. 
Table 1. Detector Parameters for the Simulated Spectra.*

\begin{tabular}{lc}
\hline Parameter & \multicolumn{1}{c}{ Value } \\
\hline Window & Moxtek AP3.3 (Manufacturer's Table) \\
Elevation & $40^{\circ}$ \\
Azimuth & $155^{\circ}$ \\
Distance & $72 \mathrm{~mm}$ \\
Area & $40 \mathrm{~mm}$ \\
Gold & $0 \mathrm{~nm}$ \\
Aluminum & $30 \mathrm{~nm}$ \\
Dead layer & $0.08 \mu \mathrm{m}$ \\
Thickness & $0.45 \mathrm{~mm}$ \\
Channel count & 4,096 \\
Zero strobe & $50 \mathrm{eV}$ \\
Energy scale & $5 \mathrm{eV} / \mathrm{ch}$ \\
Zero offset & $-479.57 \mathrm{eV}$ \\
Resolution & $124.7 \mathrm{eV}($ at $\mathrm{Mn} \mathrm{K} \alpha)$
\end{tabular}

*These correspond to the best available parameters we have for a Bruker 4040 silicon drift detector. The zero offset and resolution are from calibration with a measured copper spectrum.

of the X-ray detector or poor knowledge of the fluorescence yield. Otherwise the other line intensities were seen to fall with a standard deviation of about $5 \%$. The scaling of the Monte Carlo simulation was closer to 1.0 (1.02 versus 1.08), but this is just a result of the choice of scaling factor.

DTSA-II is also able to perform standards-based quantification of EDS spectra. When we quantify a measured
K412 spectra against dissimilar standards $\left(\mathrm{Al}_{2} \mathrm{O}_{3}, \mathrm{Mg}, \mathrm{CaF}_{2}\right.$, $\mathrm{Fe}, \mathrm{Si}$ ) using the XPP correction algorithm, we find that we can quite accurately ( $\sim 2 \%$ relative) measure each element with the exception of oxygen. We can perform the same experiment with simulated spectra and standards. The results are shown in Table 3. The advantages gained by quantifying against standards also pertain to simulated spectra. Precise knowledge of the X-ray generation physics (ionization cross section, fluorescence yield) and detector efficiency are not critical as these factors are equivalent in both the standard and the unknown. The differences between the standard and the unknown are summarized by the terms in the ZAF correction, which are to a large extent determined by the shape of the $\varphi(\rho z)$ curve. With measured spectra, the accuracy with which the mass absorption coefficient is known also influences the accuracy of the measurement. With simulated spectra, the mass absorption coefficient is the same for the simulated unknown and simulated standards.

Figure 7 compares $\varphi(\rho z)$ curves from the Monte Carlo and the XPP correction algorithm for the elements in K412. Overall the Monte Carlo and XPP produce similar $\varphi(\rho z)$ curves. $\varphi(0)$, the location of the maximum, the integrated area under the curves, and the total range are all similar. The largest inconsistency is the way the curve tapers off at large mass depth. The XPP model has an exponential tail while the Monte Carlo shows a more abrupt cut-off. Modifying the Monte Carlo to simulate randomized amounts of energy loss per trajectory step ("straggling") did not substantially change the shape of the $\varphi(\rho z)$ curve.

Table 2. This Table Compares the Intensities in the $\mathrm{K}$ or $\mathrm{K} \alpha$ Lines for Each Element in K412 as Shown in Figure 6. ${ }^{*}$

\begin{tabular}{lcccccc}
\hline Element & Oxygen & Magnesium & Aluminum & Silicon & Calcium & Iron \\
\hline Monte Carlo & $0.762 \pm 0.003$ & $0.990 \pm 0.004$ & $1.017 \pm 0.007$ & $1.008 \pm 0.003$ & $1.108 \pm 0.005$ & $1.062 \pm 0.011$ \\
Analytical & $0.796 \pm 0.003$ & $1.094 \pm 0.004$ & $1.130 \pm 0.008$ & $1.124 \pm 0.003$ & $1.151 \pm 0.006$ & $1.012 \pm 0.011$
\end{tabular}

*The number reported for each of the Monte Carlo and analytical models is the ratio of the intensity in the simulated spectrum to the intensity in the measured spectrum. The calibration of the detector is the same for both simulations and is optimal for the analytical simulation. The standard deviation of the ratios gives a measure of the precision of the simulation. If $\mathrm{O}$ is included, $\sigma($ Monte Carlo $)=$ 0.11 and $\sigma($ Analytical $)=0.12$. Without $\mathrm{O}, \sigma($ Monte Carlo $)=0.05$ and $\sigma($ Monte Carlo $)=0.06$.

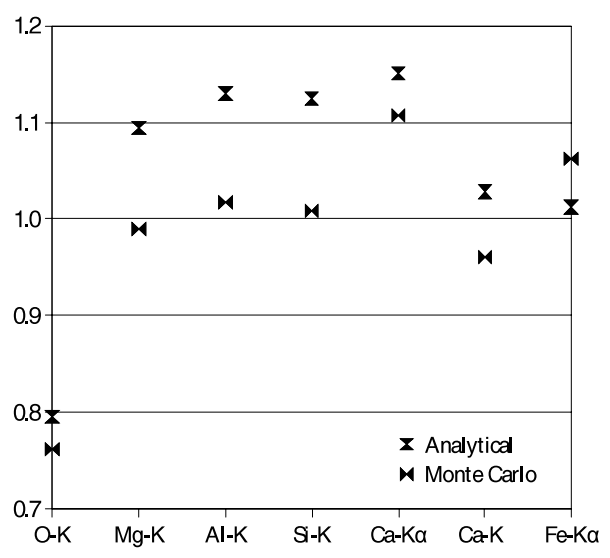


Table 3. Comparing Quantifications Performed on Measured, Analytical, and Monte Carlo Simulated Spectra.*

\begin{tabular}{lccccccc}
\hline & $\begin{array}{c}\text { Oxygen } \\
(\%)\end{array}$ & $\begin{array}{c}\text { Silicon } \\
(\%)\end{array}$ & $\begin{array}{c}\text { Magnesium } \\
(\%)\end{array}$ & $\begin{array}{c}\text { Calcium } \\
(\%)\end{array}$ & $\begin{array}{c}\text { Iron } \\
(\%)\end{array}$ & $\begin{array}{c}\text { Aluminum } \\
(\%)\end{array}$ & $\begin{array}{c}\text { Total } \\
(\%)\end{array}$ \\
\hline Measured & 44.70 & 21.43 & 11.82 & 10.82 & 7.84 & 4.77 & 101.38 \\
Analytical & 43.40 & 21.42 & 11.78 & 10.97 & 7.70 & 4.93 & 100.2 \\
Monte Carlo & 43.07 & 21.79 & 11.75 & 11.19 & 7.88 & 4.90 & 100.58 \\
Nominal composition & 43.12 & 21.38 & 11.76 & 10.99 & 7.80 & 4.95 & 100.00 \\
Measured & 3.7 & 0.2 & 0.5 & -1.5 & 0.5 & -3.6 & 1.4 \\
Analytical & 0.6 & 0.2 & 0.2 & -0.2 & -1.3 & -0.4 & 0.2 \\
Monte Carlo & -0.1 & 1.9 & -0.1 & 1.8 & 1.0 & -1.0 & 0.6
\end{tabular}

${ }^{*}$ Standard spectra were simulated for the analytical and Monte Carlo simulations. $\mathrm{Al}_{2} \mathrm{O}_{3}$ was used as a standard for $\mathrm{Al}$ and $\mathrm{O}, \mathrm{CaF}$ for $\mathrm{Ca}$, and pure elements for the remainder. The XPP algorithm (Pouchou \& Pichoir, 1991) was used to correct the fitted k-ratios. The top half of the table shows the quantified and nominal values of the composition. The bottom half shows the relative deviation from the nominal composition. Despite carefully collecting standards with good count statistics, the measured composition had an analytical total $1.4 \%$ too high. The majority of this error was in the oxygen. The analytical spectrum used the same $\varphi(\rho z)$ model to simulate and correct the k-ratios so it is not surprising that the agreement is close to count statistics limited. Note: The certified composition was normalized before performing the simulations resulting in the values reported as the nominal composition.

SRM-482 (https://srmors.nist.gov/view_cert.cfm?srm= 482) represents a range of $\mathrm{Au}-\mathrm{Cu}$ alloys from $100 \% \mathrm{Au}$ to $100 \% \mathrm{Cu}$ at nominally $20 \mathrm{wt} \%$ intervals. At $25 \mathrm{keV}$, the $\mathrm{Cu} \mathrm{K}$ and $\mathrm{L}$ and $\mathrm{Au} \mathrm{L}$ and $\mathrm{M}$ lines are all readily visible.
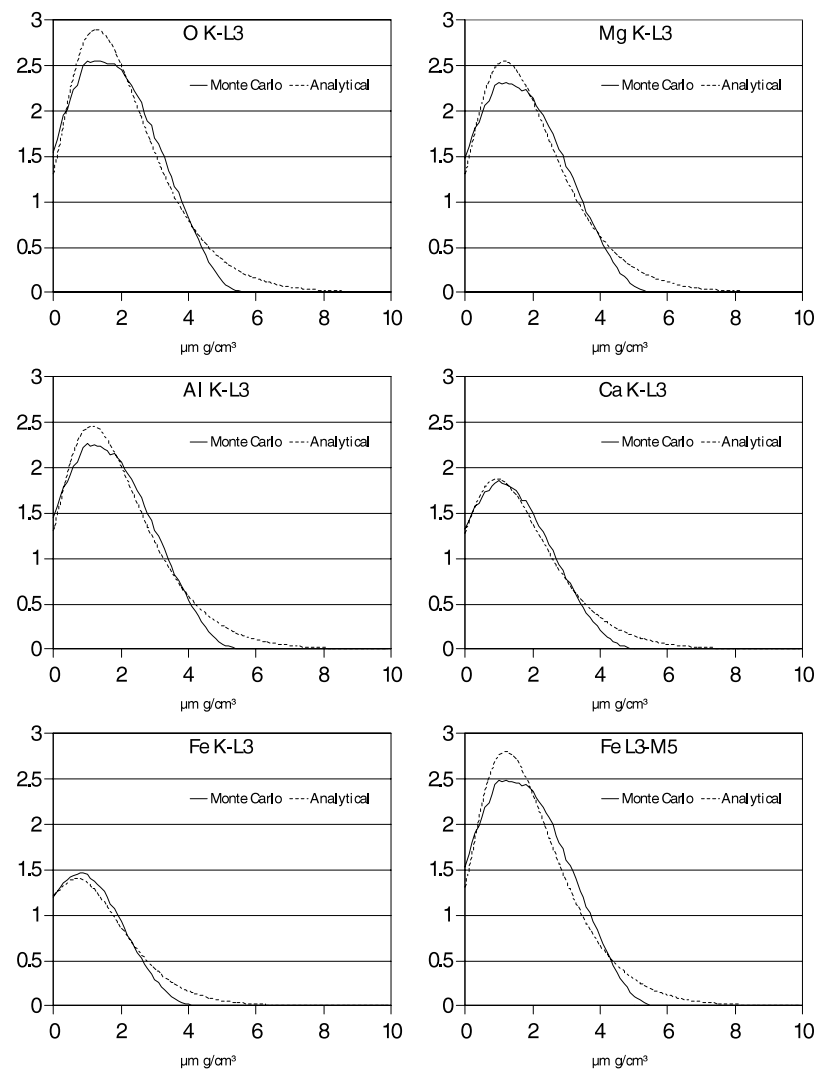

Figure 7. Comparing $\varphi(\rho z)$ curves computed with the Monte Carlo with those computed with the XPP algorithm. To facilitate comparison, the axes limits are held constant.
This makes one of the middle members of the SRM- 482 set a good material to compare $\mathrm{K}, \mathrm{L}$, and $\mathrm{M}$ lines. Figure 8 compares measured and simulated $\mathrm{Au} 60.3 \% / \mathrm{Cu} 39.6 \%$. Table 4 compares the simulated and measured intensities. The detector parameters were identical to those used to simulate the K412 spectra. While the agreement is not quite as good, it is nonetheless gratifying. Not only was the beam energy different from the K412 simulation that we used to calibrate the detector model but also the line families. For the Monte Carlo simulation, the intensity in the $\mathrm{Cu} \mathrm{K} \alpha$ is about 3\% high, the Au L lines are both about 10\% low, the $\mathrm{Au} \mathrm{M} \alpha$ line is about $10 \%$ high, and the $\mathrm{Cu} \mathrm{L}$ line is about $18 \%$ high. The analytical model agreement is generally less good with the Au M $\alpha$ line being almost 35\% too high.

While these two examples do not represent an exhaustive demonstration of the abilities of the simulations, they do suggest that the models are sufficient for many purposes. Since the models get the dose corrected intensities approximately correct, the models can be used to perform limits of detection studies. Because the Monte Carlo can model complex geometries, the models can provide insight into analysis of complex samples. Currently the models are not sufficiently accurate to perform first principles standardless analysis. The integrated analytical tools in DTSA-II facilitate comparison of measured and simulated spectra. These tools also provide a platform on which to improve our understanding of the basic physics and how it carries through to simulated spectra.

\section{Discussion}

The examples demonstrate that the DTSA-II Monte Carlo is capable of modeling gross features and is more than sufficient for answering many of the questions for which one 

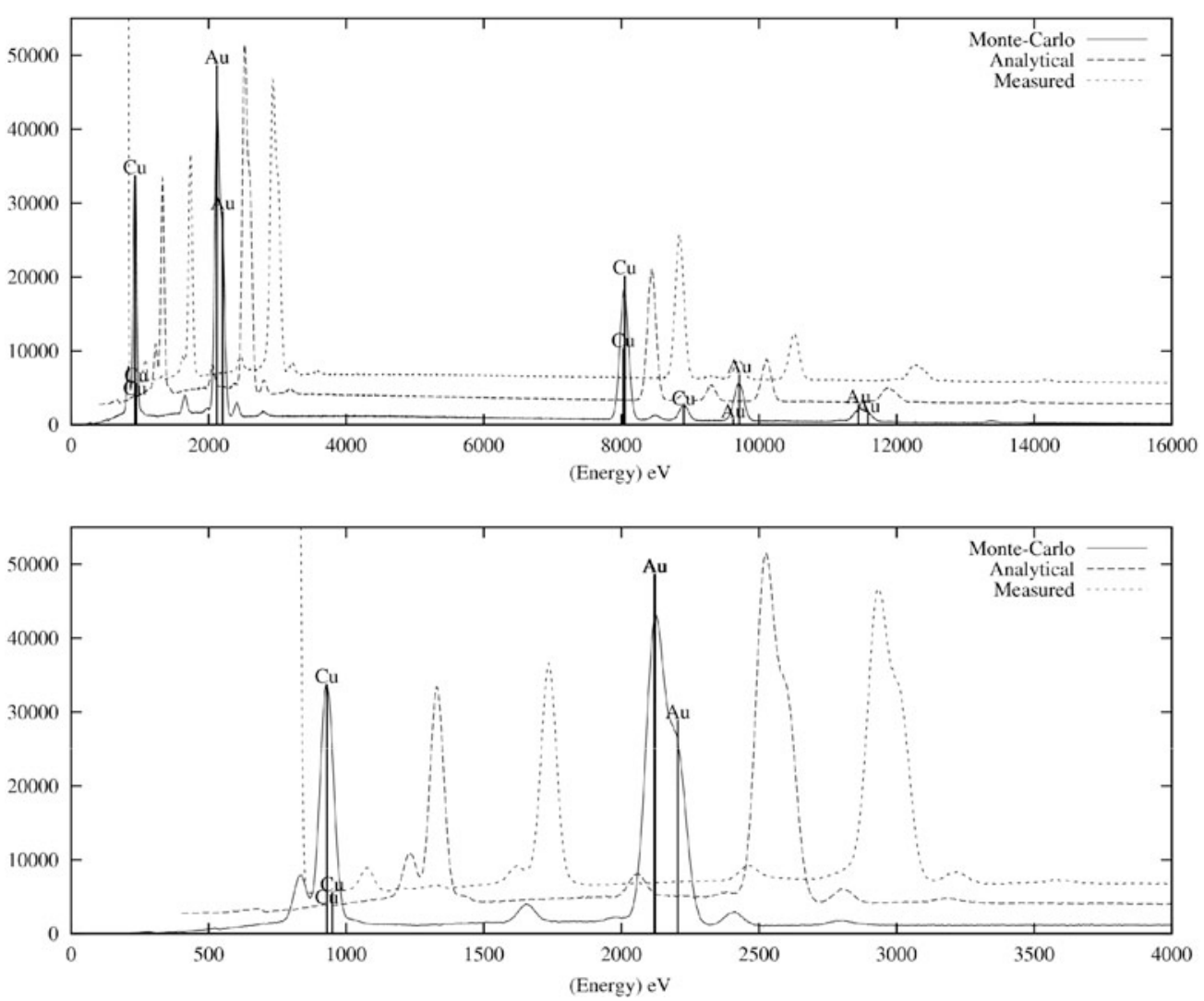

Figure 8. Simulated spectra of $60 \% \mathrm{Au}$ and $40 \% \mathrm{Cu}$ by mass to compare simulations of $\mathrm{K}$, L, and $\mathrm{M}$ lines. The spectra are staggered to facilitate comparison.

might turn to simulation. However, the examples also identified certain shortcomings. Clearly work needs to be done on weights of lines. The weights of lines are functions predominantly of the ionization cross section, the fluorescence yield, and the Coster-Konig rates. While it is not realistic to measure all these parameters for all elements, it may be possible to make progress through atomic physics calculations.

Second, the accuracy of the simulated spectra is limited by the accuracy of the detector model. The detector efficiency is most difficult to determine at low X-ray energies where many edges and strong absorption take place. It is not realistic to expect that many researchers will be able to have their detector efficiency calibrated absolutely using a calibrated X-ray source like a beam line. However, a standard like the BAM CRM (Alvisi et al., 2006) can be used to transfer the calibration from a beam line calibrated detector to an uncalibrated detector. This process involves measuring two spectra from a carefully constructed material and fitting the measured intensities to extract absolute measures of the efficiency at a handful of points. The thickness and materials of the window and detector layers are then optimized to best fit these measured points. A tool to integrate in such a calibration mechanism into DTSA-II would go far

Table 4. This Table Compares the Intensities in Each of the $\mathrm{Cu} \mathrm{K} \alpha, \mathrm{Au} \mathrm{L} \alpha$, $\mathrm{Au} \mathrm{L} \beta, \mathrm{Au} \mathrm{M} \alpha$, and $\mathrm{Cu} \mathrm{L}$ Lines with the Measured Intensity in the K or K $\alpha$ Lines for Each Element in K412 as Shown in Figure 7.*

\begin{tabular}{lccccc}
\hline Lines & $\mathrm{Cu} \mathrm{K} \alpha$ & $\mathrm{Au} \mathrm{L} \alpha$ & $\mathrm{Au} \mathrm{L} \beta$ & $\mathrm{Au} \mathrm{M} \alpha$ & $\mathrm{Cu} \mathrm{L}$ \\
\hline Monte Carlo & $1.025 \pm 0.002$ & $0.900 \pm 0.003$ & $0.899 \pm 0.007$ & $1.086 \pm 0.002$ & $1.175 \pm 0.003$ \\
Analytical & $1.081 \pm 0.002$ & $1.016 \pm 0.004$ & $0.990 \pm 0.002$ & $1.345 \pm 0.002$ & $1.264 \pm 0.002$
\end{tabular}

${ }^{\star}$ The number reported for each of the Monte Carlo and analytical models is the ratio of the intensity in the simulated spectrum to the intensity in the measured spectrum. The calibration of the detector is the same for both simulations and is optimal for the analytical simulation. The standard deviation of the ratios gives a measure of the precision of the simulation. $\sigma$ (Monte Carlo $)=0.083$ and $\sigma($ Analytical $)=0.068$. 
toward eliminating a significant source of ambiguity in interpreting the simulated spectra.

Modeling secondary fluorescence efficiently would be interesting to many including the geological community who often wonder whether a tiny peak represents a trace element or secondary fluorescence from a proximate phase. Adding secondary fluorescence should be straightforward and logically equivalent for both characteristic and bremsstrahlung radiation. Adding an additional EventListener between the X-ray generation class and the detector would allow us to track and account for X-rays that go in directions other than toward the detector.

\section{Availability of DTSA-II}

DTSA-II is available for free from the NIST Web site (http:// www.nist.gov/dtsa). The installation package includes executable modules that work on Microsoft Windows 2000/XP/ Vista, Apple OS X, Linux, and Solaris. DTSA-II requires a Java Run-time Environment (JRE) version 6 or higher (http:// java.sun.com/javase/downloads/index.jsp). The executables for DTSA-II are packaged as Java Archive (JAR) files. The epq.jar and dtsa2.jar files contain both executable modules and the source code from which these modules were compiled. The source code can be extracted from the JAR files using standard ZIP compressed file tools or using the jar, the Java archive tool. The executable modules are stored in files with a .class extension and the source code in files with a .java extension. In addition, many data files used by the library including tables of cross sections, edge and transition energies, and mass absorption coefficients are also stored within epq.jar. As a product of a U.S. government employee in the course of his appointed duties, the source code is not subject to copyright protection (Title $17 \mathrm{Sec}-$ tion 105 of the U.S. code).

\section{CONCLUSIONS}

The vision that has guided the design of DTSA-II is to provide practical answers to common microanalytical questions. The algorithms contained within DTSA-II represent a powerful set of tools for simulating, quantifying, and interpreting electron probe microanalysis measurements. DTSA-II and the libraries on which it is based have been developed and are being made available as a resource for the electronexcited X-ray microanalysis community. Furthermore, the algorithms have been factored out into an independent library to facilitate their integration into other applications. All the source code is available without limitation to facilitate reuse and to ensure that there is never any ambiguity as to how an algorithm is implemented. The source code is documented. It is hoped that this openness, which is consistent with NIST's mission of advancing the state-of-the-art in measurement science, will be appreciated by the microanalytical community as it was with the original DTSA.

\section{REFERENCES}

Acosta, E., Llovet, X. \& Salvat, F. (2002). Monte Carlo simulation of bremsstrahlung emission by electrons. Appl Phys Lett 80, 3228-3230.

Alvisi, M., Blome, M., Griepentrog, M., Hodoroaba, V.D., Karduck, P., Mostert, M., Nacucchi, M., Procop, M., Rohde, M., Scholze, F., Statham, P., Terborg, R. \& Thiot, J.F. (2006). The determination of the efficiency of energy dispersive $\mathrm{X}$-ray spectrometers by a new reference material. Microsc Microanal 12, 406-415.

Bastin, G., Dijkstra, J. \& Heijligers, H. (1998). PrOZA96: An improved matrix correction program for electron probe microanalysis, based on a double Gaussian approach. X-Ray Spectr 27, 3-10.

Bote, D. \& Salvat, F. (2008). Calculations of inner-shell ionization by electron impact with the distorted-wave and planewave Born approximations. Phys Rev A 77, 042701.

Casnati, E., Tartari, A. \& Baraldi, C. J. (1982). An empiricalapproach to K-shell ionization cross-section by electrons. Phys B: At Mol Opt Phys 15, 155-167.

Chantler, C.T., Olsen, K., Dragoset, R.A., Chang, J., Kishore, A.R., Котоснigova, S.A. \& Zucker, D.S. (2005). X-Ray Form Factor, Attenuation and Scattering Tables (version 2.1). Gaithersburg, MD: National Institute of Standards and Technology. Available at http://physics.nist.gov/ffast (accessed August 15, 2008). Originally published as Chantler, C.T. (2000). J Phys Chem Ref Data 29(4), 597-1048; and Chantler, C.T. (1995). J Phys Chem Ref Data 24, 71-643.

Cullen, D.E. (1992). Program RELAX: A code designed to calculate X-ray and electron emission spectra as singly charged atoms relax back to neutrality. UCRL-ID-110438, Lawrence Livermore National Laboratory.

Czyzewski, Z., MacCallum, D.O., Romig, A. \& Joy, D.C. (1990). Calculations of Mott scattering cross-section. J Appl Phys 68, 3066-3072.

Edgerton, R., Fiori, C., Hunt, J., Isaacson, M., Kirkland, E. \& Zaluzec, N. (1991). EMMFF V. 1.0. Available at http://www.amc. anl.gov/ANLSoftwareLibrary/EMMPDL(old)/Info/emmff.doc.

Fiori, C., Swyt-Thomas, C. \& Myкlebust, R. (2005). Desktop spectrum analyzer. Available at http://www.cstl.nist.gov/div837/ Division/outputs/DTSA/DTSA.htm.

Goldstein, J., Newbury, D., Joy, D., Lyman, C., Echlin, P., Lifshin, E., Sawyer, L. \& Michael, J. (2003). Scanning Electron Microscopy and X-Ray Microanalysis-Third Edition. New York: Kluwer Academic/Plenum Publishers.

HeinRich, K.J.F. (1986). Mass absorption coefficients for electron probe microanalysis. Proceedings of the 11th International Congress on X-ray Optics and Microanalysis, University of Western Ontario, pp. 67-119.

Henke, B., Gullikson, E. \& Davis, J. (1993). X-ray interactions: Photoabsorption, scattering, transmission, and reflection at $E=50-30000 \mathrm{eV}, Z=1-92$. At Nucl Data 54, 181-342.

Jablonski, A., Salvat, F. \& Powell, C.J. (2003). NIST Electron Elastic-Scattering Cross-Section Database-Version 3.1. Gaithersburg, MD: National Institute of Standards and Technology.

Joy, D.C. \& Luo, S. (1989). An empirical stopping power relationship for low-energy electons. Scanning 11, 176-180.

Kissel, L., Quarles, C. \& Pratt, R. (1983). Shape functions for atomic-field bremsstrahlung from electrons of kinetic energy 
$1-500 \mathrm{keV}$ on selected neutral atoms $1 \leq Z \leq 92$. Atom Data Nucl Data 28, 381-460.

Knoll, G. (2000). Radiation Detection and Measurement-Third Edition. New York: John Wiley and Sons.

Myklebust, R., Newbury, D. \& Yakowitz, H. (1976). In NBS Special Publication 460, Heinrich, K., Yakowitz, H. \& Newbury, D. (Eds.), p. 105. Washington, DC: National Bureau of Standards.

Niculae, A., Soltau, H., Lutz, G., Lechner, P., Bechteler, A., Eckhardt, R., Hermenau, K., Jaritschin, O., Liebel, A., Simsek, A., Jaritschin, O., Liebel, A., Simsek, A., Schaller, G., Schopper, F., Strüder, L., Schaller, G., Schopper, F. \& STRÜDER, L. (2008). Expanding the detector efficiency of silicon drift detectors with optimized radiation entrance window. Proceedings of 57th Annual Conference on Applications of X-Ray Analysis, Denver, Colorado, August 4-8, 2008.

Pouchou, J.-L. \& Pichoir, F. (1991). Quantitative analysis of homogeneous or stratified microvolumes applying the model 'PAP.' In Electron Probe Quantification, Heinrich, K. \& Newbury, D. (Eds.), pp. 31-75. New York: Plenum.
Press, W., Teukolsky, S., Vetterling, W. \& Flannery, B. (1992). Numerical Recipes in C-The Art of Scientific ComputingSecond Edition. New York: Cambridge University Press.

Ritchie, N.W.M. (2005). A new Monte Carlo application for complex sample geometries. Surf Interface Anal 37, 1006-1011.

Salvat, F., Fernández-Varea, J.M. \& Sempau, J. (2006). PENELOPE-2006 computer code. OECD/NEA Data Bank, Issyles-Moulineaux, France. Available at http://www.nea.fr/lists/ penelope.html.

Scott, V., Love, G. \& Reed, S. (1995). Quantitative Electron-Probe Microanalysis-Second Edition. New York: Ellis Horwood.

Seltzer, S. \& Berger, M. (1986). Bremsstrahlung energy-spectra from electrons with kinetic-energy $1 \mathrm{keV}-10 \mathrm{GeV}$ incident on screened nuclei and orbital electrons of neutral atoms with $Z=1-100$. Atom Data Nucl Data 35, 345-418.

Small, J., Newbury, D. \& Myklebust, R. (1987). Modeling of the bremsstrahlung radiation produced in pure-element targets by 10-40 keV electrons. J Appl Phys 61 (2), 459-469.

Villarrubia, J.S., Ritchie, N.W.M. \& Lowney, J.R. (2007). Monte Carlo modeling of secondary electron imaging in three dimensions. Proc SPIE 6518, 65180K 1-14. 